\title{
Oncocytoma in the deep lobe of the parotid gland; the need of a meticulous surgical approach to avoid postsurgical complications
}

\author{
Fernando PDC ${ }^{1}$, Dias DK ${ }^{1}$, Kottahachchi $\mathbf{M J}^{2}$, Thilakarathne $\mathbf{W M}^{2}$ \\ ${ }^{\prime}$ Oral and Maxillo-Facial Surgery Unit, ${ }^{2}$ Pathology Department, \\ Teaching Hospital, Karapitiya, Galle, Sri Lanka.
}

Correspondence: Dr. PDC Fernando

e-mail: dilan4fernando@gmail.com

\section{Introduction}

Salivary gland tumours arising from parotid gland affect $1: 100,000$ people world-wide, representing $2-3 \%$ of tumours of the head and neck and $1 \%$ to $1.5 \%$ of all neoplastic tumours. However only $12 \%$ of parotid tumours are located in the deep lobe $(1,2)$.

Oncocytoma (oxyphilic adenoma) is a rare benign tumour that arise mainly in salivary glands $(0.4 \%$ to $1 \%$ of parotid tumours), thyroid, parathyroid, esophagus, respiratory tract, liver, stomach, kidneys and adrenal glands mainly in sixth decade with female predominance (1). WHO classifies oncocytic neoplasms mainly into three categories: oncocytoma, the commonest, oncocytosis and focal adenomatous oncocytic hyperplasia (3). Since most parotid oncocytomas are benign, parotidectomy with preservation of facial nerve is the preferred surgical treatment (4).

\section{Case Report}

A 61-year old woman presented to Oral and Maxillofacial Unit (OMFU) of Teaching Hospital Karapitiya with a left pre-auricular swelling for four years duration. She also complained of resent onset swallowing difficulty, change in voice and sleep disturbances. There was $3 \mathrm{~cm} \mathrm{x} 4 \mathrm{~cm}$, firm, tender, well circumscribed, deep tissue attached lump resulting elevated ear-lobe pinna and obliterated posterior mandibular sulcus without facial nerve dysfunction (Figure1). Intra-orally left lateral pharyngeal wall, oropharynx and soft palate showed a moderate swelling.

Ultra-sound guided fine needle aspiration cytology (FNAC) showed benign salivary tissues, however
CT scan showed a homogenous deep lobe lesion extended to parapharyngeal space displacing carotid vessels (Figure 2). Superficial lobe of left parotid was normal. Intra-oral explorative biopsy of deep lobe through left lateral pharyngeal wall was done with guidance of CECT and under general anesthesia and it showed a benign oncocytoma. Considering the functional disability with progressive enlargement of the lesion left total parotidectomy with preservation of the facial nerve was considered for the patient.

Standard left superficial parotidectomy was performed with modified Blair incision (Figure 3a). Facial nerve landmarks were followed to identify and preserve facial nerve trunk, two main divisions and sub-divisions in anterograde manner.

Brownish red lesion in the deep lobe was noted and negative results of needle aspiration excluded the possibility of vascular origin. Facial nerve and all its branches were carefully separated from the deep lobe (Figure 3b). Tumour with the deep lobe was dissected from important surrounding structures and vessels within parotid parenchyma. Stylomandibular ligament was cut to approach parapharyngeal space for complete removal of the lesion. Complete delivery of the deep lobe including the lesion was done following blunt dissection of base of the skull structures. Histology of the removed tissue confirmed benign oncocytoma (Figure 4).

Patient recovered with House-Brackmann grade IV weakness of left facial nerve as a post-operative complication (Figure 5). Eye care and physiotherapy were provided and improvement in nerve weakness (House-Brackmann II) was observed in six months review (Figure 6). 


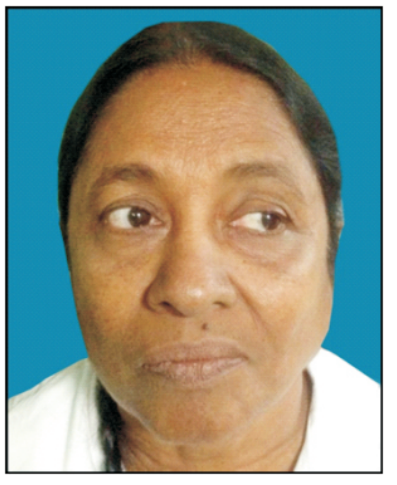

Figure 1: Pre operative view showing left parotid region swelling

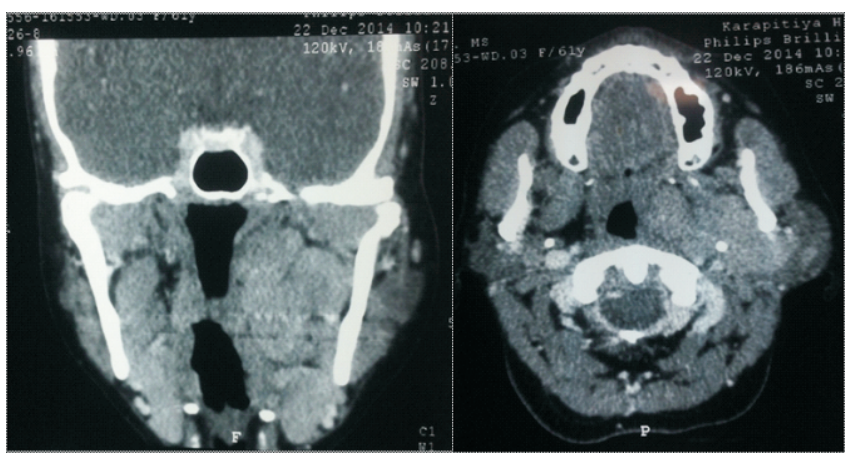

Figure 2: CT scan coronal and axial cut showing hyper dense deep lobe of left parotid gland

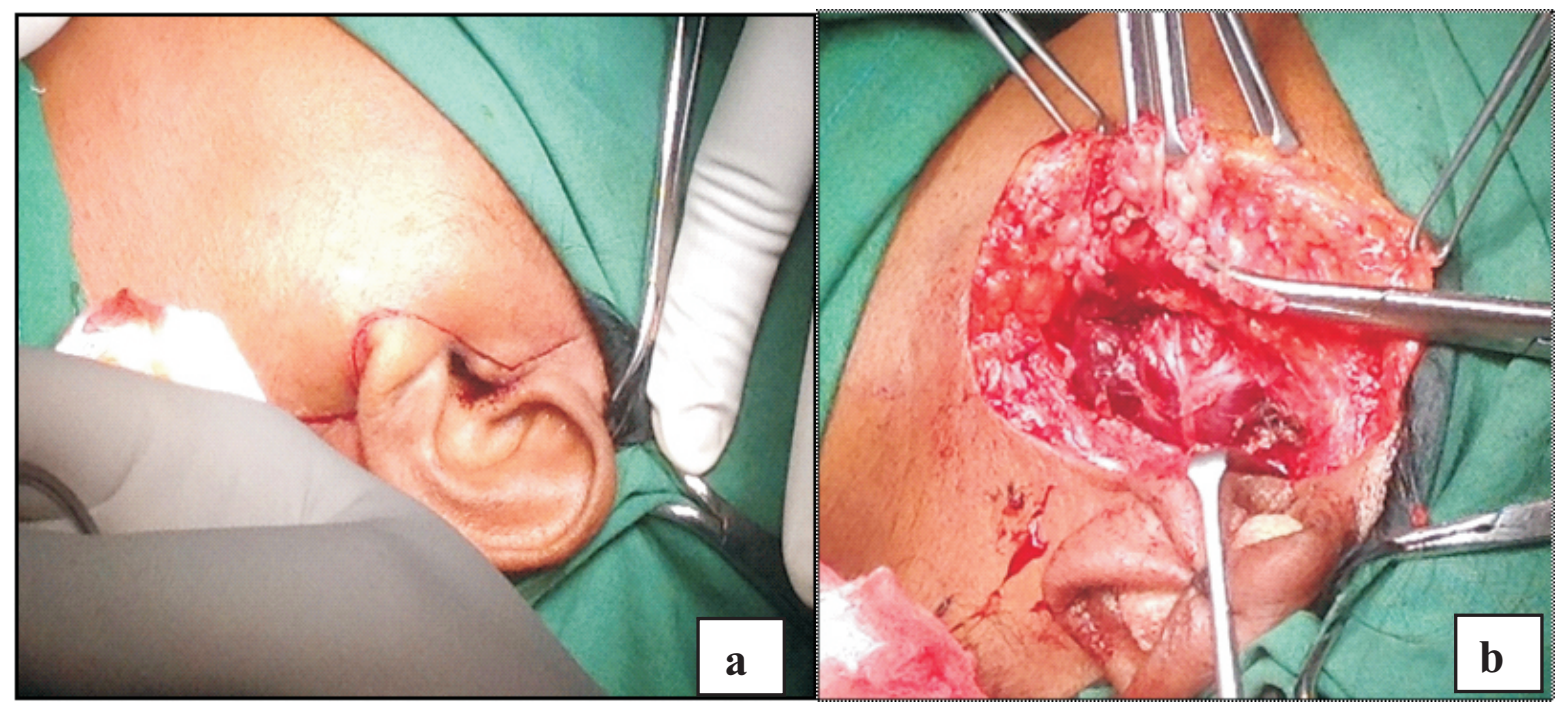

Figure 3a: Modified Blair incision to expose the left parotid region

3b: Facial nerve overlying the lesion (deep lobe-parotid) after removing the superficial parotid lobe

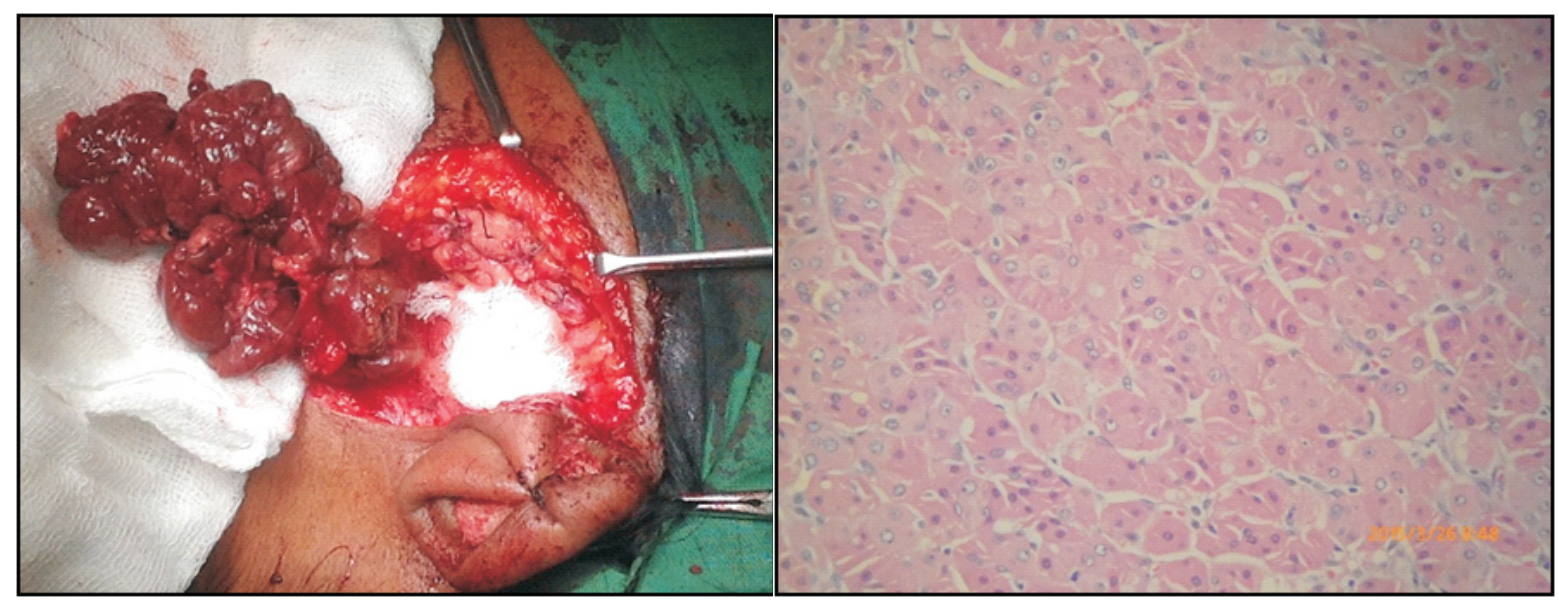

Figure 4: Excised deep lobe lesion and H \& E section showing features of oncocytoma 


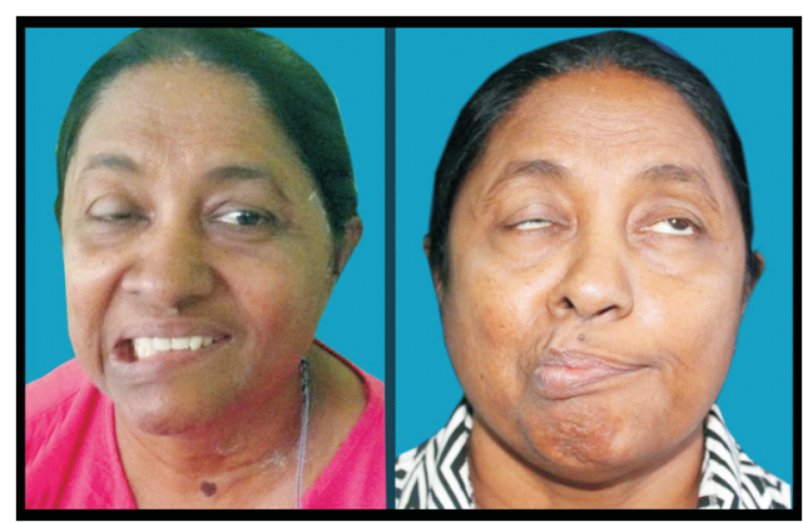

Figure 5: Postoperative view showing left facial nerve dysfunction

(House-Brackmann grade IV)

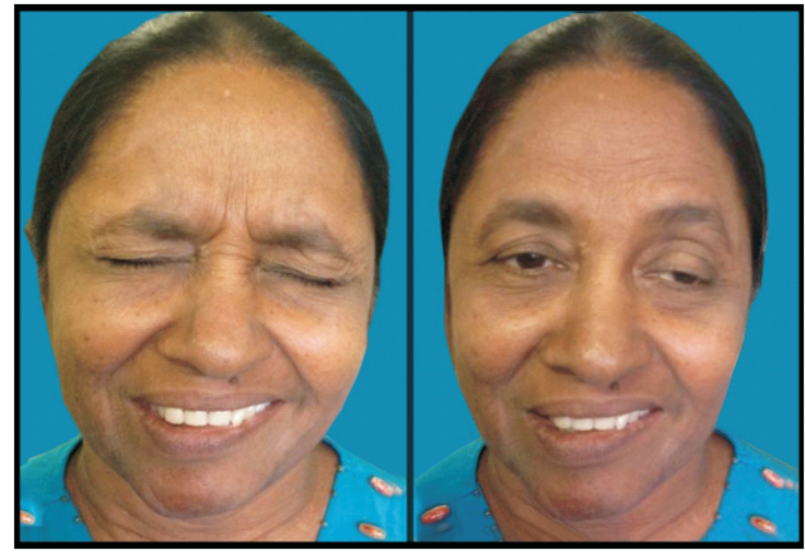

Figure 6: Recovered facial nerve function after six months (House-Brackmann grade II)

Written informed consent has been obtained from the patient to publish identifiable photographs.

\section{Discussion}

Elevation of ear lobe pinna, obliteration of the posterior mandibular sulcus and facial nerve weakness are cardinal extra oral features of a parotid tumour. However lateral pharyngeal wall and soft palate asymmetry suggest involvement of the deep lobe. Parotid duct orifice should be checked for any purulent discharge. Presence of pain, facial paralysis, rapid growth, ill-defined margins, and skin infiltration are characteristics of parotid malignancies $(3,5)$. Except facial nerve involvement and parotid duct discharge, other cardinal features were seen in this patient. Sleep apnoea and alteration of voice resulted due to the extension of a part of deep lobe into the left para-pharyngeal space (4).
Ultrasonography and FNAC are the initial investigations for salivary gland tumours (3). Biopsy is contraindicated as it may cause neoplastic seeding and nerve damages. CECT and MRI provide better visualisation, radiological diagnosis and tumour extensions during treatment planning.

Histopathologically, oncocytoma is a wellcircumscribed lesion composed of oncocytes, large uniform polygonal epithelial cells with eosinophilic granular cytoplasm and small round nuclei (5). Multi-nodular and multifocal hyperplastic variants have been described in literature (1).

No sufficient histological and ultra-structural differences among benign and malignant oncocytomas. However malignant counterpart has local invasion, destruction of surrounding structures, and regional lymphatic infiltration (5).

The widely practiced parotid surgical methods are, partial superficial parotidectomy, superficial parotidectomy, total parotidectomy with preservation of facial nerve and radical parotidectomy with or without neck dissection $(4,2)$. Since majority of oncocytomas are benign, total parotidectomy with preservation of facial nerve was the treatment of choice in this case.

Depending on the size of the deep lobe lesion surgical approach would vary. Smaller lesions can enucleate with the standard approach, however medium lesions need resection of stylomandibular ligament to approach parapharyngeal space. Large tumours need mandibulotomy at mid line, parasymphysial region or at ramus level combined with external approach $(4,2)$.

Facial nerve weakness is a major complication in parotidectomy. Transient nerve dysfunction and neuropraxia may occur due to surgical manipulation and postsurgical oedema in $8 \%-65 \%$ cases which gradually resolves in 6-18 months postsurgically. In $1 \%-3 \%$ permanent damage has been reported with axonotomesis. If accidental transection occurred it needs immediate repair of the nerve. Grater auricular and sural nerve grafts are effective when scarifying part of the main trunk.

House-Brackmann grading system is used to categorise facial nerve dysfunction (6). In this case, postoperative House-Brackmann grade $1 \mathrm{~V}$ had improved to grade II following discharge. 
Frey's syndrome is another complication in up to $4 \%-62 \%$ of post parotidectomy patients (4). Misdirection of parotid parasympathetic secretomotor fibers with superficial skin sympathetic fibers during healing is the cause. It spontaneously resolves within six months but insertion of fascia lata beneath the skin or superiorly based partial thickness sternocleidomastoid muscle flap is useful to avoid this complication. Botulinum toxin also can be used as a medical management.

In conclusion, occurrence of oncocytoma in a major salivary gland is a rare finding, and only few deep lobe parotid gland excisions were been discussed in medical literature. Moreover, advanced knowledge of clinical anatomy and surgical skills are essential for identification and preservation of the facial nerve, which is a critical factor during the surgery. Dysphagia, sleep apnoea and altered speech, which are late manifestations of a deep parotid tumour urged the treatment and the surgical outcome was satisfactory.

\section{References}

1. Stafford RE, Ray M, Schubert W. Benign Oncocytoma of the Deep Lobe of the Parotid Gland. J Oral Maxillofac Surg 1999; 57: 346-50.

2. Zhang SS, MaDQ, GuoCB, el al. Conservation of salivary secretion and facial nerve function in partial superficial parotidectomy. International Journal of Oral Maxillofacial Surgery 2013; 42: 868-73.

3. Sungur N, Akan IM, Ulusoy MG, et al. Clinicopathological evaluation of parotid gland tumours: A retrospective study. JCraniofacial Surg 2002; 13: 26-30.

4. Patel M. Surgical techniques for parotid and submandibular glands and branchial cysts. In: Booth PW. Schendel SA, Hausamen JE. Maxillofacial Surgery. $2^{\text {nd }}$ ed. V(1). Missouri: Churchill Livingstone;1999: p680-95.

5. Eveson J. Salivary Gland disorders. In: Warnakulasuriya S, Thilakaratne WM. Oral Medicine and Pathology. A guide to diagnosis and management. Jaypee 2014: p235-51.

6. House JW, Brackmann DE. Facial nerve grading system. Otolaryngol Head Neck Surg 1985; 93: 146-7. 